\title{
ON A COUNTEREXAMPLE OF GAROFALO - LIN FOR A UNIQUE CONTINUATION OF SCHRÖDINGER EQUATION*
}

\author{
WILL Y. LEE \\ Department of Mathematical Sciences \\ Rutgers University-Camden, \\ Camden, N.J.08102
}

\begin{abstract}
Garofalo and Lin have given a counterexample for which unique continuation fails for the Schrödinger equation

$$
-\Delta u+\frac{c}{|x|^{2+\varepsilon}} u=0, \varepsilon>0 .
$$

Their counterexample consists of a Bessel function of the third kind $\mathrm{K}_{\mathrm{V}}(|\mathrm{x}|)$ with the restriction that $v$ cannot be an integer. In this note we have removed the restriction.

Key Words: Schrödinger Equation, Unique Continuation, Bessel Function of the Third Kind, Inverse Square Potential
\end{abstract}

AMS Subject Classification : 35A07, 35L05.

Consider the following Schrödinger equation:

$$
-\Delta u+V u=0
$$

Garofalo and Lin [ 1] have shown that $V=c /|x|^{2}$, the inverse square potential, is optimal for unique continuation of (1). Indeed they have given a counterexample for which unique continuation fails once the square inverse potential is replaced by $\mathrm{c} / \mid \mathrm{x}^{2+\varepsilon}$ for any $\varepsilon>0$ [1: pp. 265-266]. Their counterexample is given by

$$
u(x)=|x|^{-(n-2) / 2} K_{(n-2) / \varepsilon}\left(\frac{2 \sqrt{c}}{\varepsilon}|x|^{-\varepsilon / 2}\right)
$$

* Received: October 1990, Revised: January 1991 
with the restriction that $(n-2) / \varepsilon$ cannot be an integer for any $\varepsilon>0$

In this note we show that this restriction is unnecessary. We use the same notations as in [1].

Theorem: For the following Schrödinger equation,

$$
-\Delta u+\frac{c}{|x|^{2+\varepsilon}} u=0 \text { in } B_{1}
$$

where $\mathrm{B}_{1}$ is a unit ball in $\mathrm{R}^{\mathrm{n}}$, we have a radial solution given by

$$
u(x)=|x|^{-(n-2) / 2} K_{(n-2) / \varepsilon}\left(-\frac{2 \sqrt{c}}{\varepsilon}|x|^{-\varepsilon / 2}\right)
$$

where $\mathrm{K}_{(\mathrm{n}-2) / \varepsilon}$ is a Bessel function of the third kind for any real number $(\mathrm{n}-2) / \varepsilon$ and $\varepsilon>0$.

Proof. Since $\Delta_{\mathrm{x}} \mathrm{u}=\mathrm{u}_{\mathrm{rr}}+\frac{\mathrm{n}-1}{\mathrm{r}} \mathrm{u}_{\mathrm{r}}+\frac{1}{\mathrm{r}^{2}} \Delta_{\theta} \mathrm{u}$, a radial solution of (2) must satisfy

$$
r^{2} u^{\prime \prime}(r)+(n-1) r u^{\prime}(r)-c r^{-\varepsilon} u(r)=0,0<r<1 .
$$

It is known that $I_{v}(z)$ and $I_{-v}(z)$, Bessel functions of imaginary argument, satisfy the differential equation [ $3:$ p. 77 ]

$$
z^{2} \frac{d^{2} u}{d z^{2}}+z \frac{d u}{d z}-\left(z^{2}+v^{2}\right) u=0
$$

Notice from (4) and (5) that $I_{v}(z)$ and $I_{-v}(z)$ (when $v$ is an integer, see equation (9) for the definition) cannot be a solution of (4) because of the coefficient n-1 of $u^{\prime}(r)$ in (4). Thus we look for a solution of a form $z^{\alpha} I_{ \pm v}\left(\beta z^{y}\right)$, where $\alpha, \beta$, and $\gamma$ are constants to be determined. An easy computation reveals that $\left.z^{\alpha} I_{ \pm v}\left(\beta z^{y}\right)\right)$ satisfies:

$$
\begin{aligned}
z^{2} \frac{d^{2}}{d z^{2}}\left(z^{\alpha} I_{ \pm v}\left(\beta z^{y}\right)\right)+ & (1-2 \alpha) z \frac{d}{d z}\left(z^{\alpha} I_{ \pm v}\left(\beta z^{y}\right)\right) \\
- & \left(\gamma^{2} \beta^{2} z^{2 y}+\gamma^{2} v^{2}-\alpha^{2}\right)\left(z^{\alpha} I_{ \pm v}\left(\beta z^{y}\right)\right)=0 .
\end{aligned}
$$


Comparison of (6) with (4) shows that

$$
\alpha=-(\mathrm{n}-2) / 2, \beta=-2 \sqrt{\mathrm{c}} / \varepsilon, \gamma=-\varepsilon / 2 \text {, and } v=(\mathrm{n}-2) / \varepsilon \text {. }
$$

Define the third kind of Bessel function $\mathrm{K}_{\mathrm{v}}(\mathrm{z})$ according to Watson [ 3: p. 78] by

$$
\begin{array}{ll}
K_{v}(z)=\frac{\pi}{2} \frac{I_{-v}(z)-I_{v}(z)}{\sin v \pi}, & v \neq \text { integer } \\
K_{n}(z)=\lim _{v \rightarrow n} K_{v}(z), \quad v=\text { integer. }
\end{array}
$$

Then $K_{v}(z)$ is defined for all real values of $v$. Conjunction of equations (4)-(9) yields the solution (3) to Schrödinger equation (2). This completes the proof.

\section{Remark.}

Since $K_{v}(r) \sim r^{-1 / 2} e^{-r}$ for all $v$ as $r \rightarrow \infty$ [3: p.202], $K_{v}(r)$ vanishes of infinite order as $r \rightarrow \infty$. Consequently unique continuation of Schrödinger equation (2) fails for any $\varepsilon>0$, which implies that $V=c / \mid x^{2}$, the inverse square potential, is optimal for unique continuation of solutions of Schrödinger equation (1).

\section{References}

[1] Garofalo, N. and Lin, F.H., Monotonocity Properties of Variational Integral. $A_{p}$ Weights and Unique Continuation, Indiana Univ. Math. Jour., Vol. 35, No. 2 (1986), 245-268.

[2] Garofalo, N. and Lin, F.H., Unique Continuation for Elliptic Operators: A Geometric-Variational Approach, Comm. Pure \& Appl. Math., Vol. XL (1987) 347-366.

[3] Watson, G.N., A Treatise on the Theory of Bessel Functions, Cambridge Univ. Press (1966). 


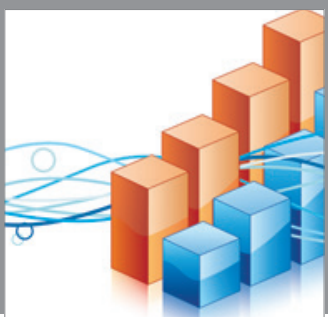

Advances in

Operations Research

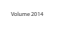

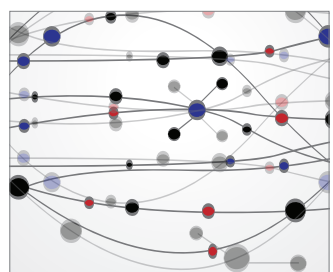

\section{The Scientific} World Journal
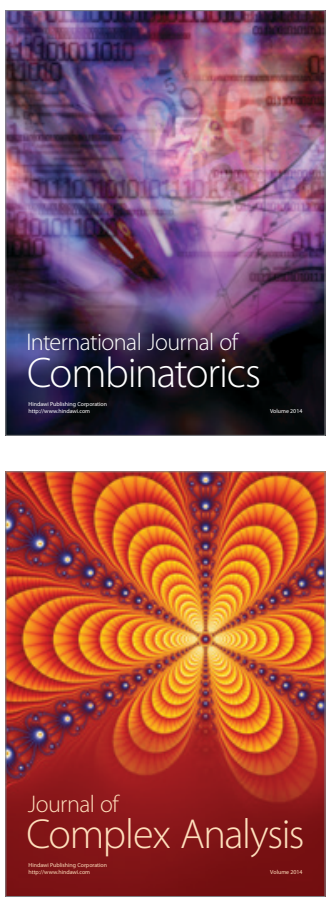

International Journal of

Mathematics and

Mathematical

Sciences
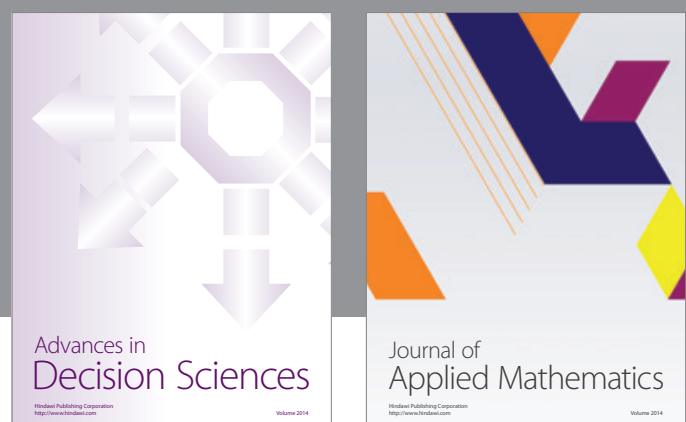

Journal of

Applied Mathematics
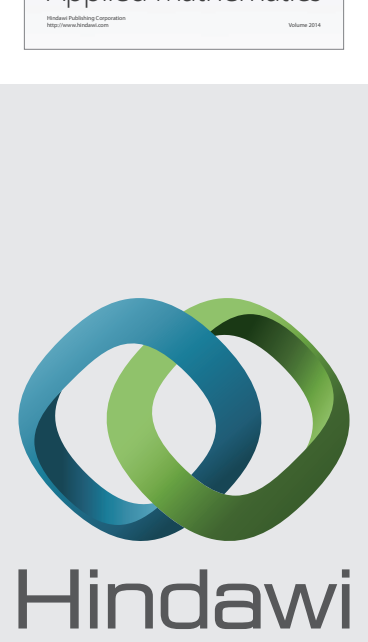

Submit your manuscripts at http://www.hindawi.com
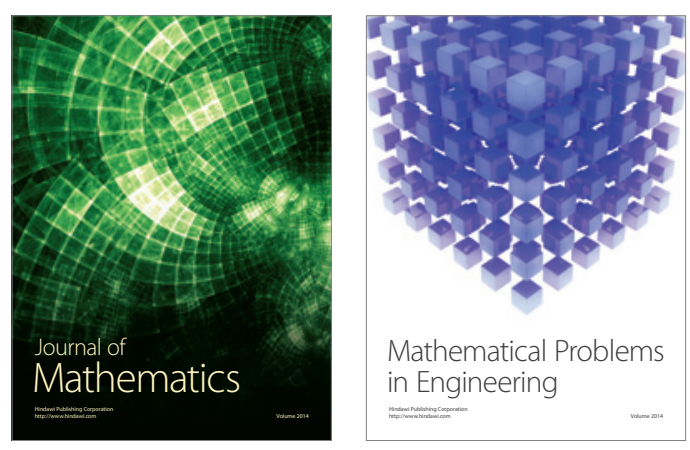

Mathematical Problems in Engineering
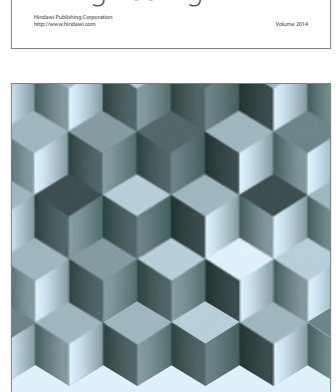

Journal of

Function Spaces
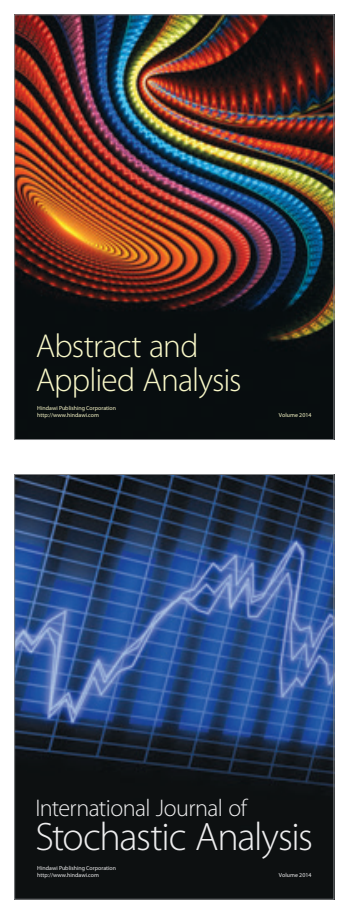

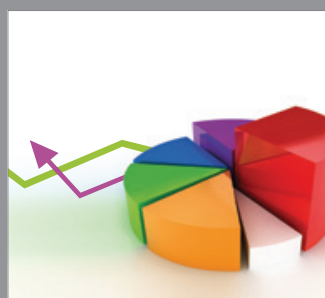

ournal of

Probability and Statistics

Promensencen
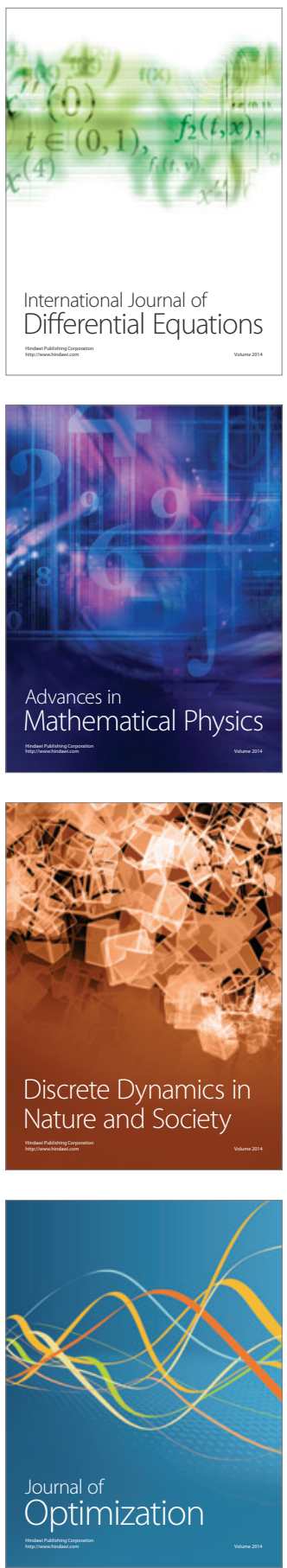